\title{
Género, medioambiente y equidad: Tres conceptos claves en la gestión de Recursos Humanos del presente y futuro.
}

\author{
doi: 10.33264/rpa.202101-01 \\ Bárbara Acuña-Jujihara, Cecilia Baeza-Correa \\ Escuela de Humanidades UNIACC \\ Facultad de Humanidades y Ciencias Sociales UNIACC
}

\section{Resumen}

El desarrollo del presente ensayo se enmarca en una reflexión acerca de la gestión de recursos humanos en las empresas y organizaciones de nuestro país, poniendo el énfasis en tres grandes desafíos que están lejos de ser considerados como logrados: (1) Igualdad de género, (2) Gestión ambiental sostenible y (3) Equidad social. Estos desafíos se enmarcan en exigencias emanadas de acuerdos internacionales firmados y ratificados por nuestro país, en relación a los tres ámbitos presentados, que se vinculan por una parte a los derechos laborales de las y los trabajadores, como también a la competitividad de las organizaciones a nivel internacional. Estos desafíos suelen aparecer en términos formales pero pocas veces desarrolladas de manera real al interior de las empresas y organizaciones y suelen responder a presiones internacionales asociados a exigencias de certificación. En las siguientes páginas se presentan antecedentes vinculados a cada uno de los desafíos, como también experiencias exitosas a modo de referencia.

Palabras clave: Gestión de Recursos humanos, Género, Medioambiente y Equidad

\section{Abstract}

The development of this essay is framed in a reflection on human resources management in companies and organizations in our country, emphasizing three major challenges that are far from being considered as achieved: (1) Gender equality, (2) Sustainable environmental management and (3) Social equity. These challenges are framed within the requirements emanating from international agreements signed and ratified by our country, in relation to the three areas presented, which are linked on the one hand to the labor rights of workers, as well as to the quality of organizations that allow their competitiveness at an international level. These challenges usually appear in formal terms but are rarely developed in a real way within companies and organizations and usually respond to international pressures associated with certification requirements. The following pages present background information related to each of the challenges, as well as successful experiences as a reference.

Keywords: Human Resources, Gender, Environment and Equity Management 


\section{Introducción}

En el presente ensayo se abordan tres desafíos que enfrenta, actualmente en las organizaciones medianas y grandes en Chile, la gestión de recursos humanos. Estos desafíos emergen de los cambios sociales, tanto globales como locales, a los cuales las organizaciones deben poder dar unarespuesta oportuna y competitiva que les permita mantenerse vigentes (Suárez-Campasa, Álvarez-Medinaby, \& VásquezTorres, 2020).

Los tres temas elegidos, además de ser temas contingentes, dialogan con los denominados indicadores de desempeño sustentable o ESG por sus siglas en inglés (Environmental, Social and Governance) y que apuntan efectivamente a la relevancia que en el sector de las inversiones se da a los temas ambientales, sociales y de gobierno de las empresas. Los puntajes de ESG indican la calificación que los analistas de Bloomberg otorgan al grado de transparencia y responsabilidad delos informes de la compañía sobre los tres pilares de las estrategias ESG, el desempeño y las actividades relacionadas (Carrillo Flórez, y otros, 2020, pág. 82). De acuerdo a algunos estudios, existe una relación positiva entre aquellas compañías que trabajan en el marco de la ESG y el valor de la firma y de sus calificaciones crediticias (Devalle, Fiandrino, \& Cantino, 2017; Carrillo Flórez, y otros, 2020).

Ahora bien, en una sociedad como la chilena, caracterizada por el doble estándar, la ambigüedad y la inconsistencia, que tiene su correlato en el mundo empresarial y, especialmente, en la gestión de recursos humanos (Perez Arrau, 2008, pág. 84) la gestión del cambio organizacional adquiere un cariz particular, ya que respecto de los tres temas que se abordarán a continuación, los cambios requeridos responden fundamentalmente a presiones externas a las organizaciones y quecomienzan a pujar en materia de la sostenibilidad organizacional, por lo que el cambio aparece como un camino obligado, que resulta mucho más complejo de gestionar (Goncalves, 2020).

En este sentido, resulta fundamental que las organizaciones puedan adaptarse a estos nuevos requerimientos del mundo actual y abordar estas temáticas desde la gestión de recursos humanos, considerando que las personas son el diferencial competitivo que propician y sostienen el éxito de la organización; son su principal ventaja competitiva dentro de un mundo globalizado, inestable y cambiante en el que existe una competencia feroz (Chiavenato, 2009, pág. 4).

\section{Desarrollo}

A continuación, y para profundizar en lo presentado, revisaremos de manera independiente cada uno de los desafíos identificados para este ensayo.

\section{Desafío 1: Igualdad de género}

Uno de los desafíos globales que deben abordar las empresas actualmente dice 
relación con la gestión de recursos humanos respecto de la igualdad de género. Este concepto, en términos laborales tiene su primera expresión en el Tratado de Roma (1957), que abordó la igualdad de trato y salario para mujeres y hombres por la realización del mismo trabajo. Un hito muy relevanteen esta materia se enmarca en la Cuarta Conferencia Mundial de las Mujeres realizada en Beijing (1995) en cuyos postulados se deja en evidencia la existencia de un sistema de género que determina las relaciones sociales y que tiene como resultado la desigualdad entre hombres y mujeres (Rey Aramendía, 2020, pág. 334). Sin embargo, a pesar de la evidencia disponible y del transcurso de los años, aún la igualdad de género sigue siendo un tema pendiente en la sociedad en general y en la esfera laboral en particular.

La brechas de igualdad de género en el espacio laboral se expresan principalmente en las diferencias de salarios, dificultades en el acceso a un empleo formal, conciliación laboral y vida familiar y la poca participación de las mujeres en cargos directivos o gerenciales (Montalvo Romero, 2020) todos estos temas que afectan directamente a la gestión de $\mathrm{RRHH}$, incluyendo temas que revisten una complejidad mayor como son la discriminación en el ámbito de la crianza yel aumento de casos de abuso y acoso sexual (Castillo, 2020).

Si bien, existen publicaciones que cuestionan el aporte de la inclusión de las mujeres en los equipos de trabajo, aludiendo a que tienen mayores tasas de absentismo y rotación más alta (Mayo, 2006), este tipo de argumentos puede ponerse en tensión debido a que pasa por alto variables sociales estructurales que terminan incidiendo en un comportamiento laboral diferenciado para hombres y mujeres, debido a que son estas últimas las que terminan corriendo con el costo total de las labores asociadas a la reproducción y al cuidado (Comunidad Mujer, 2017). Otros elementos estructurales que afectan el desempeño laboral de las mujeres, se asocian a las peores condiciones de empleo (entre un $9 \%$ y un $6 \%$ por debajo de los hombres) en materia de cualificación y autonomía (demandas cognitivas, control del ritmo de trabajo y formación) y en discrecionalidad y perspectivas (tipo de contrato y perspectivas de permanencia y promoción), según revela un estudio realizado a partir de los datos disponibles de la Encuesta Europea de Condiciones de Trabajo, que realiza la Fundación Europea para la Mejora de las Condiciones de Vida y del Trabajo de la Unión Europea (Eurofound), y aunque si bien se señala que existenalgunos ámbitos donde la calidad del empleo es mejor para las mujeres, como es el caso decalidad del tiempo de trabajo, señalan que puede deberse a que las mujeres deben ausentarsemás por las tareas de cuidado que ejercen con mayor frecuencia que sus colegas varones (Antón \&Muñoz de Bustillo Llorente, 2020).

A pesar de lo anterior, es decir de las inequidades estructurales en el desempeño laboral con las que cargan las mujeres, existen estudios que destacan y promueven las ventajas de su inclusión en el mundo laboral. De acuerdo a la noticia publicada el 
08 de octubre del 2020 en el sitio web investing.com, denominada "Inclusión social, igualdad de género, diversidad: Hay 2 ETF paraesto" " se mencionan estudios que empíricamente avalan el aporte de la inclusión de las mujeres almercado laboral, afirmando por ejemplo que "La aceleración del crecimiento del PIB de Estados Unidos en el marco de un aumento de la participación de la fuerza laboral femenina podría sumar 5,87 billones de dólares a la capitalización del mercado mundial en 10 años". Añadiendo que los beneficios sociales y económicos de tener compañías más inclusivas no solo aplica a EEUU, sino que a todo el mundo.

En términos de las exigencias laborales y en consideración a sus implicancias para el área de RRHH,resulta interesante considerar que, en el mes de septiembre del año 2020, se ingresó un proyecto de ley con el propósito de modificar el Código del Trabajo para establecer medidas de transparencia para las empresas en materia de igualdad de remuneraciones entre hombres y mujeres. El argumento detrás de este proyecto se funda en el señalamiento de la Organización Internacional del Trabajo (OIT) que indica que "La igualdad de remuneración es un derecho humano reconocido al que tienen derecho todos los hombres y mujeres" (Oelz, Olney, \& Tomei, 2013, pág. 4). En términos estadísticos, el mismo proyecto consigna que, actualmente en Chile, las mujeres asalariadas reciben ingresos $29,3 \%$ menores a los de los hombres. Como sanción, al incumplimiento de la transparencia de salarios por género, se considera una multa de 100 a 1000 UTM, junto con la imposibilidad de ser contratada por la Administración del Estado (El Mostrador Braga, 2020).

Esta acción va en línea con la necesidad de que los Estados generen políticas laborales efectivas ${ }^{2}$ con enfoque de género que permeen la relación en el reclutamiento y contratación, en el desarrollo laboral y en el término de la relación contractual (Montalvo Romero, 2020, pág. 15).

En este sentido, resulta crucial que las organizaciones planifiquen la incorporación de la transversalización de género, de manera que la gestión de recursos humanos pueda abordar los múltiples desafíos que la igualdad de género implica: erradicación de la brecha salarial; equidad enlos puestos de poder; evaluación del "techo de cristal" y del "piso pegajoso"; mismos costos laborales para hombres y mujeres según labores de reproducción y cuidado; por mencionar algunos. Para ello, resulta fundamental que las áreas de recursos humanos se hayan capacitado y cuenten con perspectiva de género que les permita, efectivamente, poder avanzar en saldar la deuda histórica hacia las mujeres.

${ }^{1}$ Disponible en: https://es.investing.com/analysis/social-inclusion-gender-equality-diversity-there-are--2-etfs-for-that-200439007

${ }^{2}$ Cabe destacar que en Chile existe la Ley $\mathrm{N}^{\circ} 20.348$, que Resguarda el Derecho a la de Igualdad de Remuneraciones, pero a

más de diez años de su entrada en vigor, no ha disminuido la brecha salarial entre hombres y mujeres

Revista Pensamiento Académico de la Universidad UNIACC

Vol. 4 N¹, 2021 


\section{Desafío 2: Gestión ambiental sostenible}

Junto a lo anterior, otro gran desafío global que deben enfrentar actualmente las organizaciones y en particular sus áreas de RRHH, corresponde a los temas de gestión ambiental. La relevancia que está ganando el tema ambiental a nivel organizacional se observa en acciones concretas como fue el acuerdo firmado por más de 26 empresas y organizaciones -entre ellas Adidas, Unilever, Nestlé,ABN-Amro- con el propósito de levantar una declaración conjunta para impulsar que se legisle para hacer obligatoria la Debida Diligencia en Derechos Humanos y Medio ambiente en la Unión Europea (UE) (Pérez, 2020).

Si bien, la incorporación de la temática de la sostenibilidad como un quehacer propio del área de Recursos Humanos no se encuentra ampliamente extendida, según lo demuestra el estudio realizado por el Centro para Organizaciones Efectivas, que al consultar a los ejecutivos de recursos humanos sobre el rol del área en los temas de sostenibilidad de su empresa, "solo el 3\% reconoce tener la responsabilidad primaria en este ámbito $y$, de forma paralela, únicamente el 1\% dice que debería tener la responsabilidad primaria" (Roca, 2014). Por su parte, Edward Lawler y Susan Morhman, en el estudio publicado en la revista MIT Sloan Management Review, indican que "Un buen punto de partida es que recursos humanos tome un papel importante en la construcción de lasostenibilidad en todo lo que hace como función y en todos los procesos que controla" (Roca, 2014).

En esta misma línea, el artículo denominado Prácticas de recursos humanos que impactan la estrategia de sostenibilidad ambiental (2019) presenta por cada ámbito del trabajo del área de RRHH, el aporte que puede hacer en materia ambiental, así respecto de la Planeación del talento: indican que pueden garantizar que el talento requerido para ejecutar la estrategia desostenibilidad esté disponible en el presente y en el futuro; Diseño de cargos y del trabajo: debe diseñar todos los cargos a partir de tareas y metas vinculadas con el desempeño ambiental que se quiere alcanzar. Además en esta línea se encuentran acciones que aportan a la mitigación de impacto ambiental como es el trabajo flexible; Atracción y permanencia de talento: en la que se puede avanzar mediante la creación de una oferta de valor asociada a la sostenibilidad ambiental, considerando además la relevancia que dan a los temas ambientales en el quehacer laboralgeneraciones como la de los Millennials (León, 2020); Reclutamiento y selección de talento: para avanzar en la línea de la sostenibilidad ambiental es fundamental contar con empleados/as comprometidos/as y sensibles con dichos temas. En este sentido, estos procesos resultan claves; Aprendizaje y desarrollo de talentos: la formación temas ambientales es un elemento central paraimplementar una estrategia de sostenibilidad en las organizaciones; Administración del conocimiento y la cultura organizacional: busca cambiar la cultura organizacional, de modo que laorganización y sus empleados/as 
tengan profundamente adheridos los valores con los que apoyarán la sostenibilidad a largo plazo; Gestión del desempeño: es la que permitirá alcanzar los objetivos ambientales trazados por la organización; finalmente, la Compensación: puede servir de herramienta para promover la actitud proambiental (Gutiérrez-Rua, PosadaGarcía, Gonzalez- Perez, \& Gonzalez-Perez, 2019, págs. 15-20).

En este contexto se aprecia cómo el Área de RRHH, ejerciendo sus funciones habituales, puede jugar un rol clave en la adaptación de las organizaciones a las exigencias ambientales del presente y futuro, que cada día se profundizan más.

\section{Desafío 3: Equidad social}

Finalmente, en términos locales, las medianas y grandes empresas chilenas enfrentan un desafío importante que ha sido puesto de manifiesto tras la Revuelta social del 18 de octubre de 2019. El malestar social expresado en las movilizaciones ciudadanas iniciadas ese día, puso sobre la palestra la desigualdad social imperante y junto con ello la desconexión existente entre quienes detentanla propiedad de las empresas (y sus planas mayores) y la realidad que viven las y los trabajadores de dichas organizaciones. Da cuenta de esto las propias palabras de Alfredo Enrione, presidente del Círculo de Directores7, quien señala "Nuestra clase media es mucho más pobre y frágil que la que desde la elite percibimos" (Weissman, 2020). En términos numéricos, esta distorsión de la realidad se expresa, por ejemplo, en la percepción de la élite sobre la estructura de clases en el país, señalando que el $25 \%$ es clase baja, el $57 \%$ es clase media y el $18 \%$ es clase acomodada. Los datos reales indican que: $77 \%$ es clase baja, solo el $20 \%$ clase media y un escaso $3 \%$ es clase acomodada (Weissman, 2020). Sobre la afiliación al sistema de Isapres, las élites estiman que el 39\% de la clase media y el $18 \%$ de la clase baja están afiliados a estas. La realidad es que el $8 \%$ y $0 \%$, lo están. Finalmente, respecto del valor de la vivienda la élite sobrestima el valor en comunas de nivel socioeconómico medio y bajo (NSE), estiman que en un sector medio una casa cuesta poco más de un tercio de lo que vale una en Lo Barnechea, cuando en realidad una casa en Lo Barnechea vale 30 veces más que una en Quinta Normal (Weissman, 2020).

En este marco, en que la desigualdad económica es posible en un contexto donde la libertad de las empresas está por encima de la vida digna para las personas, donde las reglas del mercado permiten acumular riqueza sin límite, y los estados lo legalizan y lo fomentan resulta relevante poner sobre el tapete que la equidad es un factor de prosperidad a largo plazo (Calvo Palomares, Portet Tebas, \& Bou Pastor, 2016, pág. 4).

De este modo, bajo la premisa que reducir la desigualdad es imperante - por ello se expresa en el Objetivo de Desarrollo Sostenible $\mathrm{N}^{\circ} 10^{8}$ - el desafío para recursos 
humanos es grande, ya que por una parte puede ser un área con participación clave en el aporte de las empresas a la reducción dela desigualdad, y por otra parte, debe procurar la relación armónica y comprometida de losequipos de trabajo para el logro de los objetivos de la empresa. En palabras de Beatriz Sánchez Guitián, Directora General de Fundación máshumano: “Las empresas más competitivas y sostenibles son conscientes de que su equipo humano es clave para anticiparse, innovar, moverse con agilidad y generar confianza en todos sus públicos de interés" (Compromiso Empresarial, 2020). Coincidente con esta mirada, en el plano más local, Mariana Bargsted, Directora del Magíster en Habilidades Directivas de la Universidad Adolfo Ibáñez, señalando que los directivosde RRHH deben volver a tener un rol asesor estratégico y permanente en la cúpula directiva, recordando con insistencia que el motor, y a la vez fin último, del crecimiento económico, son las personas (Bargsted, 2019).

En particular, respecto del quehacer del área de recursos humanos, Mariana Bargsted, en elmismo artículo, identifica seis puntos en los cuales RRHH puede jugar un papel clave en la disminución de la desigualdad. Estos puntos son: 1) Salarios: la autora indica que "es responsabilidad de las áreas de Recursos Humanos insistir e implementar un piso y un techo a los salarios que permita tener organizaciones más equitativas"; 2) Beneficios: propone que sean aquellos que efectivamente requieran las y los trabajadores (flexibilidad laboral, recompensas económicas, tiempos de descanso, etc.); 3) Extensión de la jornada laboral: indica que 40 horas laborales son razonables y que resulta imperante que las tareas asignadas puedan ser efectivamente desarrolladas en ese tiempo. Añade que el contar con más tiempo para hacer actividades propias (tiempo familiar, ocio, etc.) puede aportar en la disminución de los altos niveles de estrés laboral que existen, que según información publicada en septiembre de 2019 en el sitio web trabajando.com el 82\% de las y los chilenos ha presentado algún cuadro de estrés laboral el último año (Trabajando.com, 2019); 4) Procesos de reclutamiento y selección de personas: señala que se debe avanzar en la elaboración de estándares técnicos y éticos, al interior de las organizaciones de manera de evitar una discriminación arbitraria y exclusión de colectivos sociales específicos, junto con ello resulta importante evitar el "doble estándar" en la selección de personal en que se argumenta el uso de herramientas sofisticadas, pero que en el paso inicial de la convocatoria operan sesgos de discriminación por edad, género, comuna de residencia entre otros(Perez Arrau, 2008, págs. 84-85). En esta misma línea, Bargsted incluso añade que debiesen existir leyes y normas que obliguen a auditar la calidad de los procesos como ocurre en otros países; 5) Programas de desarrollo de talento: indica que es importante revisarlos, puesto que en lasorganizaciones se va replicando una desigualdad interna con la sobre calificación de algunaspersonas respecto de la nula oferta para otras, lo que a su vez repercute en el mejor desempeño organizacional limitando el potencial del 
resto de las y los trabajadores; finalmente, en el punto 6) Formación para el liderazgo: considerando que las habilidades de liderazgo deben ser formadas, hace hincapié en se promuevan aquellas relativas a la capacidad de auto-conciencia, de empatía y de auto-regulación, habilidades y sensibilidad a la diversidad y a la dignidad de las personas, orientación al servicio y al bienestar, acompañando a la orientación al logro y la efectividad(Bargsted, 2019).

Según lo expuesto se observa cómo el área de RRHH, resulta ser un área de gestión clave, que puede aportar a una distribución más equitativa de los recursos de las organizaciones, relevando la importancia de las y los trabajadores para la sostenibilidad y logro de los objetivos de las mismas.

\section{Conclusiones}

En conclusión, se observa que el área de recursos humanos resulta clave para poder avanzar y dar una respuesta organizacional que esté acorde con las necesidades y demandas de los nuevos tiempos. En este sentido, tener en vista experiencias exitosas a nivel internacional, generar normativas internas, pautas e indicadores que permitan monitorear los avances en estos temas, son medidas básicas que recursos humanos no puede dejar de hacer.

Es así como, en la medida que se le dé urgencia al interior de las organizaciones al avance en estos temas, podemos esperar que se logre la sostenibilidad y bienestar organizacional, considerando que, según lo expuesto, las normativas nacionales e internacionales están avanzando con paso decidido en los temas de igualdad de género, gestión ambiental sostenible y equidad social.

Resulta interesante también considerar, desde este enfoque, la posibilidad de que las empresas se posicionen como organizaciones estratégicas que permitan avanzar de manera efectiva en ladeuda histórica frente a los temas de igualdad de género y equidad social, como abordar elementos que permitan aportar a la mitigación de la crisis climática actual.

Identificando la relevancia que tiene la esfera del trabajo en la sociedad chilena, es posible aspirar a que las empresas puedan ser organizaciones aliadas para el desarrollo social y que finalmente el aporte y avances que introduzcan en materia de derechos humanos permee la estructura social, pasando a ser un ente socializador de estos importantes desafíos sociales.

Finalmente, es importante destacar que resulta fundamental contar con registros y hacer seguimiento de las medidas que las Áreas de RRHH tomen en estas materias, de manera de poder contar en el mediano plazo con referentes y con mediciones claras que den cuentan del valor que dichas prácticas añadieron al quehacer de la organización.

Revista Pensamiento Académico de la Universidad UNIACC

Vol. $4 \mathrm{~N}^{\circ} 1,2021$ 


\section{Referencias}

Antón, J.-l., y Muñoz de Bustillo Llorente, R. (9 de Agosto de 2020). La brecha de género en eltrabajo va más allá del salario. El Mostrador, pág. Braga.

Bargsted, M. (22 de Noviembre de 2019). Cómo la Gestión de Recursos Humanos puede aportar al cambio social. Noticias UAI.

https://noticias.uai.cl/columna/como-la-gestion-de-recursos-humanos-puedeaportar-al- cambio-social/

Calvo, R., Portet Tebas, J., y Bou Pastor, M. (2016). La equidad social como elemento esencial para el desarrollo local. En J. A. Márquez, J. M. Jurado Almonte, \& F. J. Pazos García, Desarrollo local en territorios de fronteras (págs. 1-11). Universidad de Huelva.

Carrillo, M., Castaño, J., Castillo , J., Cepeda, V., Melo, N., Peñaloza, P., y otros. (2020). Sostenibilidad en la Cadena de Valor. Informe del Laboratorio Empresarial Cesa 2020-I. Editorial CESA.

Castillo, F. (4 de Octubre de 2020). DMujeres: Desigualdad de género en el mundo del trabajo. El Mostrador.

Chiavenato, I. (2009). Gestión del Talento Humano. McGraw-Hill.

Compromiso Empresarial. (20 de Octubre de 2020). Nace el Índice de Humanización de la Organización para evaluar a las empresas. www.compromisoempresarial.com/rsc/2020/10/indice-humanizacionorganizacion-evaluar-empresas/

Comunidad Mujer. (Marzo de 2017). Mujer y trabajo: Uso del tiempo y la urgencia por compartir las tareas domésticas y de cuidado. http://www.comunidadmujer.cl/biblioteca-publicaciones/wpcontent/uploads/2017/04/BOLETIN-38-marzo-2017-baja_vf.pdf

Devalle, A., Fiandrino, S., y Cantino, V. (2017). The Linkage between ESG Performance and Credit Ratings: A Firm-Level Perspective Analysis. International Journal of Business and Management , 12 (9), 53-65.

El Mostrador Braga. (2020, 14 de septiembre). Proyecto de ley busca fijar medidas de transparencia para que las empresas cumplan con la igualdad de remuneraciones.

Goncalves, V. (2020). HCMBOK - The Human Change Management Body of Knowledge: Gestión del Cambio Organizacional - El Factor Humano en el Liderazgo de 
Proyectos. SimplissimoLivros Ltda.

Gutiérrez-Rua, J., Posada-García, M. D., Gonzalez-Perez, M.-A., \& Gonzalez-Perez, M.A. (2019). Prácticas de recursos humanos que impactan la estrategia de sostenibilidad ambiental. Innovar: Revista de Ciencias Administrativas y Sociales , 11-24.

León, J. (2020). ¿Por qué los 'millennials' son un reto para las empresas? BBVA. https://www.bbva.com/es/millennials-reto-empresas/

Mayo, M. (2006). La gestión de la diversidad. En J. Bonache, \& Á. Dirección de Personas: Evidencias y perspectivas para el siglo XXI (págs. 371-395). T Prentice Hall.

Montalvo Romero, J. (2020). El Trabajo desde la Perspectiva de Género. Revista de la Facultad de Derecho, 1-19.

Oelz, M., Olney, S., \& Tomei, M. (2013). Igualdad salarial - Guía introductoria. OIT.

Perez, G. (2008). Gestión de recursos humanos en el contexto social y cultural chileno. Asian Journal of Latinamerican Studies , 65-95.

Pérez, V. (2020, 16 de octubre). Respeto a los derechos humanos y al medio ambiente: los grandes retos del mundo empresarial. El Mostrador.

Rey Aramendía, M. (2020). Mainstream de género. Eunomía. Revista en Cultura de la Legalidad, 331-341.

Roca, J. (2014, 22 de diciembre). La estrategia de recursos humanos al servicio de la sostenibilidad. Compromisos empresarial. RSC.

Suárez-Campasa, M. S., Álvarez-Medinaby, M. T., \& Vásquez-Torres, M. d. (2020). La gestión de cambio organizacional: variables asociadas para una implementación exitosa. Ciencia, Economía y Negocios, 4 (1), 69-83.

Trabajando.com. (23 de Septiembre de 2019). El 82\% de los chilenos ha presentado algún cuadro de estrés laboral el último año. https://blog.trabajando.cl/el-82-delos-chilenos-ha-presentado-algun-cuadro-de-estres-laboral-el-ultimo-ano/

Weissman, I. (2020, 19 de Octubre). El informe hecho por la élite sobre la élite y que los dejó tremendamente incómodos. El Mostrador , pág. El Semanal. 


\section{Bárbara Acuña Jujihara}

MBA (c) en Administración y Dirección de Empresas USACH. Lic. en Sociología, Diplomada en Transversalidad de Género e Institucionalidad: Un enfoque teórico y práctico, Postítulo en Ecología Social y Sustentabilidad, Diplomada en Investigación Social del Cuerpo y las Emociones. Dedicada al trabajo de gestión universitaria con enfoque de derechos, sustentabilidad y pertinencia social con los territorios. En términos de investigación ha dirigido y participado en la realización de diversos estudios de investigación cuantitativa y cualitativa principalmente referidos a la participación ciudadana, género y evaluación de Programas municipales y gubernamentales, a nivel comunal, regional y nacional. En la actualidad, ejerce la jefatura de la Unidad de Vinculación con el Mundo Público y Social, de la Vicerrectoría de Vinculación con el Medio de la Universidad de Santiago de Chile.

https://orcid.org/0000-0002-5411-5896

\section{Cecilia Baeza Correa}

Antropóloga y Magíster en Estudios de Género y Cultura, mención Ciencias Sociales. Actualmente cursando el doctorado en Estudios Americanos en la Universidad de Santiago de Chile. Dedicada a la docencia universitaria y a la investigación vinculadas a la Antropología del género y Antropología de la salud, líneas teóricas a partir de las cuales ha profundizado en la experiencia subjetiva de mujeres en relación a las siguientes temáticas: diada salud/enfermedad, sexualidad y envejecimiento. El desarrollo de sus investigaciones, se ha construido desde una perspectiva feminista. Integrante del núcleo académico de la Facultad de Humanidades y Ciencias Sociales de la Universidad UNIACC.

https://orcid.org/0000-0002-9584-2563 